\title{
RELACIÓN ENTRE LAS FORMAS DE UN VERBO SUPLETIVO: EL CASO DEL GALLEGO, COMPARADO CON EL DE OTRAS LENGUAS IBERORROMÁNICAS
}

\author{
ILDIKó SZIJJ \\ Universidad Eötvös Loránd, Budapest
}

The relations among the forms of a suppletive verb: the case of Galician, in comparison with other Ibero-Romance languages

The verb 'go' (Spanish, Portuguese, Gatician ir, Catalan anar) is a suppletive verb in the Iberian romance languages. The roots and their distribution in the paradigm are similar in standard Spanish, Portuguese and Galician, but rather different in Catalan. There is a diachronic variaton and a synchronic difference between these languages in the 4th and 5 th person of the present

- indicative mood. The variants can also be observed in dialects: according to the Galician Linguistic Atlas (Atlas linguiistico galego), the root can vary in the Galician linguistic territory in the previous two forms and in the 5 th person of the imperative mood. These three forms within the paradigm are related in that they are the "iruly" arbyzotonic forms in the three present tenses and they bave the same morpbological structure.

\section{Introducción}

El verbo IR es uno de los más irregulares tanto en gallego, como en las otras lenguas iberorrománicas y la mayoría de las románicas, por ser un verbo supletivo, con diferentes radicales. Sin embargo, los étimos concretos y la distribución de los radicales pueden diferir según las lenguas o según los dialectos.

Si postulamos que en el paradigma los elementos afines presentan el mismo radical, la comparación de la distribución de los radicales en las diferentes lenguas nos permitirá hacer algunas observaciones sobre la relación de los elementos del paradigma.

En gallego, la lengua normativa prescribe determinadas formas, y con ello cierta combinación de los radicales, pero en el dominio lingǘstico podemos encontrar otras combinaciones. Las variaciones tienen lugar en la cuarta y quinta personas del presente de indicativo y en el imperativo plural, por ello centraré mi atención en estas formas.

Empezaré esbozando la distribución de los radicales en el paradigma del verbo IR en las lenguas iberorrománicas, con algunas referencias al francés y al italiano. A continuación observo las variantes dialectales de las formas correspondientes a 
VAMOS, VAIS e ID en gallego (aprovechando los datos del Atlas lingüistico galego'). Compararé brevemente la variạción del radical del gallego con el mismo fenómeno del catalán (teniendo en cuenta los datos de la obra La flexió verbal en els dialectes catalans de Alcover y Moll2).

\section{Formas normativas del verbo IR en las lenguas iberorrománicas}

\subsection{El verbo IR en español y portugués}

Empezamos con estas dos lenguas por ser las más conocidas en el grupo de las lenguas iberorrománicas.

En español y en portugués el verbo IR tiene formas con diferentes radicales: esp. ir, voy, fui, port. ir, vou, fui. Los verbos latinos de los cuales se formó el verbo son IRE 'ir', VADERE 'ir, dar pasos' y ESSE 'ser'.

En las principales lenguas románicas, excepto el rumano, el verbo IR es supletivo, pues nació de la confluencia de VADERE y otro(s) verbo(s). Los verbos español y portugués son especiales, en comparación con el francés, el italiano y el catalán, pues en la configuración de los elementos del paradigma no interviene *ANDARE, ya que estas dos lenguas tienen un verbo independiente formado a partir de dicho étimo, andar. Por el contrario, entre los étimos del verbo ir del español y del portugués consta el verbo IRE (este étimo entra también en la formación del paradigma del verbo francés, dando origen a las formas del futuro y del condicional, jïrai, jirais, pero en español y portugués hay más formas procedentes de IRE, como ir, yendo, iba, etc.) y también ESSE. La distribución de las formas derivadas de VADERE dentro del paradigma difiere también un tanto de la francesa o de la italiana (esp. vamos; cf. fr. nous allons, it. andiamo).

En el paradigma del verbo español vienen de IRE el infinitivo (ir), por consiguiente el futuro y el condicional (iré, iría), el pretérito imperfecto de indicativo (iba), el gerundio (yendo), el participio (ido) y el imperativo plural (id). Las formas portuguesas son parecidas: ir, irei, iria, ia, indo, ido, ide. Además en portugués procede del mismo verbo también la quinta persona del presente de indicativo: ides.

En las dos lenguas provienen de VADERE las formas del presente de indicativo (excepto ides del portugués), las del presente de subjuntivo y el imperativo singular: esp. voy, vas, etc., vaya, vayas, etc., ve; port. vou, vais, etc., vá, vás, etc., vai.

Proceden de ESSE las formas de los tiempos de tema de perfecto (esp. fui, fuiste, fue, etc., fuera, fueras, etc., fuese, fueses, etc., port. fui, fora, fosse, etc.). La confluencia de los dos verbos 'ir' y 'ser' se produce en el uso perfectivo por la confusión entre los verbos de sentido direccional y de sentido locativo ('ir a algún lugar' y 'encontrarse en algún lugar').

Así, entre el español y el portugués tan solo hay diferencia en la distribución de las formas en un punto: en español en la quinta persona del presente de indicarivo tenemos

1 Instituto da Lingua Galega, Atlas lingüistico galega, Vol. I, 2, Morfoloxía Verbal, La Coruñà, Fundación "Pedro Barrié de la Maza, Conde de Fenosa", 1990.

2 Antoni M. ALCOVER - Francesc de B. MOLL, "La flexió verbal en els dialectes catalans", in: Anuari de l'Oficina Romànica de Lingüistica i Literatura. Vol. 2, 73-184, vol. 3, 6-166, vol. 4, 9-104, vol. 5, 9-72, 1929-1933. 
vais, en portugués ides. Por ello en español no hay correspondencia entre la forma del indicativo y la del imperativo: ind. vais - imp. id (cf. port. ind. ides - imp. ide). En el caso de ambas lenguas podemos afirmar que la forma del imperativo plural es regular, si consideramos su relación con el infinitivo: esp. ir - id (como cantar - cantad).

La explicación que se da para la distribución de las formas es que no se conservan las formas monosilábicas del verbo IRE ( $i s$, it), así como las formas que presentaban hiato (eo), pues también hubieran dado una forma monosilábica. Sin embargo se conservó (además del infinitivo) id, pues para expresar un mandato es adecuada una forma breve ${ }^{3}$.

En la lengua antigua podían existir otras formas: en español imos, ides aún eran formas algo usadas en el período clásico ${ }^{4}$, en portugués en el indicativo existían paralelamente vamos e imos, en el imperativo plural ide (también $i$ ) y vais.

En español existe una forma coloquial para el imperativo plural: veis. De esta manera se unifica el radical de todas las formas del presente de indicativo, del presente de subjuntivo e imperativo.

El verbo ir en español tiene otra peculiaridad relacionada con el imperativo: la cuarta persona posee una forma de imperativo específica, aparentemente indicativa, lo cual es excepcional, pues la cuarta persona en el imperativo en todos los demás verbos españoles adopta la forma del subjuntivo, p. ej. cantemos, hagamos. En el caso del verbo ir en el subjuntivo tenemos vayamos, en el imperativo vamos < VADAMUS, por lo tanto históricamente subjuntivo. Al mismo tiempo, también puede aparecer la forma vayamos con función de imperativo.

En resumen, en el plano sincrónico vemos las siguientes relaciones en el paradigma: el radical del infinitivo coincide con el del gerundio, el del participio y el del pretérito imperfecto de indicativo (ir, yendo, ido, iba), lo cual es normal si tenemos en cuenta que en el sistema verbal estas formas constituyen un grupo compacto (p. ej. en un verbo irregular: hacer, haciendo, haita, el participio puede ser diferente, como en este caso, bacer, hecho). El mismo radical aparece también en la forma id del esp. e ide del portugués, en el imperativo plural. Por otro lado, existe una relación estrecha entre las formas del presente de indicativo, del presente de subjuntivo y del imperativo singular (voy, vaya, ve), lo cual también está en consonancia con las pautas generales de la conjugación española. Por último tienen el mismo radical el pretérito indefinido de indicativo, el pretérito imperfecto de subjuntivo (fui, fuera / fuese; en portugués fora es pretérito pluscuamperfecto de indicativo); en portugués también el futuro de subjuntivo, como en los verbos irregulares (bice, hiciera / hiciese, port. fiz, fizera, fizesse, fizer).

${ }^{3}$ Manuel ALVAR - Bemard POTTIER, Morfologia bistórica del español, Madrid, Gredos, 1993, 228. +Ramón MENÉNDEZ PIDAL, Manual de gramática bistórica española, Madrid, Espasa-Calpe, 198016, 304; Paul M. LLOYD, Del latin al español. Madrid, Gredos, 1993, 475.

5 Joseph HUBER, Gramática do Português Antigo, Lisboa, Fundação Calouste Gulbenkian, 1986, 225; José Joaquim NUNES, Compêndio de Gramática Histórica Portuguesa, Lisboa, Clássica Editora, $1989^{9}, 338$.

- Leonardo GÓMEZ TORREGO, Hablar y escribir correctamente, Gramática normativa del español actual II, Madrid, Arco/Libros, 2006, 496. 


\subsection{El verbo IR en gallego}

Trato ahora el verbo gallego, que es muy semejante al español y al portugués. Tiene como origen los mismos verbos, IRE, VADERE y ESSE. Al mismo tiempo en gallego también existe un verbo independiente, andar, procedente de *ANDARE.

La distribución de los radicales en el paradigma de IR es como en las otras dos lenguas. En el punto en que hay discrepancia entre el español y el portugués, es decir, en la quinta persona del presente de indicativo, la normativa gallega prescribe ides, como en portugués, así la forma es coherente con la del imperativo, ide. El gallego diverge de las otras dos lenguas en la cuarta persona del presente de indicativo: la forma normativa es imos (cf. esp./port. vamos). Esto significa que en el presente de indicativo las formas arrizotónicas presentan un radical diferente del de las formas tizotónicas, distribución que recuerda la observable en francés e italiano: vou, vas, vai, imos, ides, van. Esta relación intraparadigmática parece más natural que la del portugués, pues las formas rizotónicas y arrizotónicas también divergen en otros verbos, p. ej. esp. cuento pero contamos, port. agrido pero agredimos, etc.

De la misma manera que en español, según la normativa gallega la cuarta persona del imperativo tiene una forma especial, que se distingue de la del subjuntivo: la forma del subjuntivo es vaiamos, mientras que en el imperativo la forma específica es vamos, que coincide con la española.

\subsection{El verbo IR en asturiano y aragonés}

En asturiano aparecen también los mismos tres radicales, procedentes de IRE, VADERE y ESSE. La distribución de las formas es como en español: presente de indicativo voi/vo, vas, va, vamos, vais, van; imperativo ve, dii; presente de subjuntivo vaya/ vaiga, etc.; pretérito imperfecto de indicativo diba, etc.; futuro diré, etc.; condicional diria, etc.; pretérito indefinido fui, etc.; pretérito pluscuamperfecto de indicativo y pretérito imperfecto de subjuntivo fuera/fuere/fora/fore, etc.; infinitivo dir; gerundio diendo; participio dio

En aragonés los étimos del verbo son también IRE, VADERE y ESSE. La distribución de las formas es algo diferente de la del español. En el presente de indicativo el paradigma es boi, bas, ba, imos, iz, ban, por lo tanto en las formas arrizotónicas el étimo es IRE, como en gallego. Otro punto interesante del paradigma es el pretérito imperfecto de subjuntivo, pues su radical no proviene de ESSE, sino de IRE: ise, ises, ise, isenos, isez, isen. Así no hay relación formal entre este tiempo y el pretérito indefinido de indicativo: fue, fues, fue, etc. (Encontramos la misma relación en otros verbos, p. ej. dar. pretérito indefinido die, pretérito imperfecto de subjuntivo dase, o sea, este último se forma a partir del radical del infinitivo; por el contrario, en el verbo ser el radical del pretérito imperfecto de subjuntivo coincide con el del pretérito

7 Academia de la Llingua Asturiana, Gramática de la Llingua Asturiana, Uviéu, Academia de la Llingua Asturiana, 19992, 209. 
indefinido: fuese - fue.) En los otros tiempos el radical es como en español: pretérito imperfecto de indicativo iba, etc.; presente de subjuntivo baiga, etc.; futuro iré, etc.; condicional irba, etc.; infinitivo ir, gerundio indo; participio iu/ito. En el imperativo, la segunda y la quinta personas tienen el mismo radical que en español, bes, ir. En la cuarta persona del imperativo la forma es imos, homónima de la forma del presente de indicativo (mientras el presente de subjuntivo es baigamos) ${ }^{8}$.

\subsection{El verbo correspondiente a IR en catalán}

Hemos dejado el catalán para el final, por tener un paradigma bastante diferente de los anteriores. Los étimos del verbo son VADERE y *ANDARE, como en francés y en italiano. El verbo IRE interviene indirectamente en la configuración del paradigma, pues ejerció influencia en algunas formas: aniré, aniria?.

La distribución de las formas catalanas es semejante a la del italiano (cat. vaig, anem. ind., vagi, anem subj.; it. vado, andiamo ind., vada, andiamo subj.), mientras que en francés hay algunas diferencias (cat. vagi; it. vada; cf. fr. que jaille). Vienen de VADERE las formas rizotónicas del presente de indicativo (vaig, vas, va, van) y del presente de subjuntivo (vagi, vagis, vagi, vagin), así como el imperativo singular (ves). Proceden de *ANDARE (o alguna variante) las formas arrizotónicas de los tiempos anteriores (anem ind./subj., aneu ind./subj./imp.), el pretérito imperfecto de indicativo (anava, etc.), el pretérito indefinido de indicativo (aní, etc.), el pretérito imperfecto de subjuntivo (anés, etc.), el infinitivo (anar), el gerundio (anant) y el participio (anat).

Al mismo tiempo en catalán ciertas formas del verbo anar se usan también para formar tiempos compuestos. Entre estos el más común es el perfecto perifrástico del indicativo, en que aparecen las formas del presente de indicativo de anar. En este uso únicamente encontramos las formas procedentes de VADERE: vaig / vas / va / vam / vau / van cantar. Así en lugar de anem y aneu, formas del paradigma cuando el verbo se usa como verbo de movimiento, aparecen vam, vau cuando el verbo funciona como auxiliar. Vemos lo mismo en el pasado perifrástico del subjuntivo: vagi / vagis / vagi / vàgim / vàgiu / vagin cantar (vàgim, vàgiu en lugar de anem, aneu). Las formas del indicativo, cuando el verbo funciona como auxiliar, tienen variantes formadas a partir del mismo radical: vares, vàrem, vàreu, varen, donde la $r$ surgió por analogía de la sexta persona del pretérito de todos los verbos, p. ej. amaren, prengueren, partiren etc. ${ }^{10}$

En la lengua antigua, en el pasado perifrástico las formas de la cuarta y quinta personas eran anam, anats (formas antiguas, antecesoras de anem, aneu del actual catalán central), que más tarde sufrieron nivelación analógica por efecto de las otras personas ${ }^{11}$.

\footnotetext{
8 Francho NAGORE, Gramática de la Lengua Aragonesa, Zaragoza, Mira Editores, 19895, 178-179.

${ }^{9}$ Francesc de B. MOLL, Gramàtica bistòrica catalana, València, Universitat de València, 2006, 225. ${ }^{10}$ Antoni BADIA I MARGARIT, Gramàtica bistörica catalana, València, Tres i Quatre, 1981, 371. 11 Idem.
} 


\section{Las variantes dialectales de las formas correspondientes a VAMOS, VAIS e ID en gallego \\ 2.1. Método y datos}

A continuación mi objetivo será observar las variantes dialectales de las formas del verbo ir del gallego. Para ello aprovecho las informaciones aportadas por el Attas lingüistico galego.

Observando el paradigma del presente de indicativo y del imperativo del verbo español, portugués y gallego, hemos visto que las formas correspondientes a VAMOS, VAIS e ID presentan variación en el radical respecto a la forma de las otras personas. Existen las siguientes combinaciones: esp. voy, vas, va, vamos, vais, van; id ; port. vou, vais, vai, vamos, ides, vão; ide; gallego normativo vou, vas, vai, imos, ides, van; ide. Las tres distribuciones se explican fácilmente: en español se distingue del resto de las formas la del imperativo; en portugués discrepan las dos quintas personas; en gallego normativo las tres formas que en los verbos regulares son arrizotónicas. Me interesa averiguar si existen otras combinaciones posibles. Para ello observo los datos dialectales del gallego, puesto que el Atlas ofrece suficiente material para conocer los paradigmas dialectales.

Según el Atlas únicamente existen variantes dialectales procedentes de ambos étimos en la cuarta y quinta personas del presente de indicativo y en el imperativo plural. Esto significa que en los demás tiempos verbales existen variantes dialectales, pero el radical es el mismo que el de la forma normativa. P. ej. el pretérito imperfecto de indicativo tiene las variantes $i b a$, $i a$, iña, pero no existen formas dialectales como *vadía, etc., de la misma manera tampoco hay variación de radical en el presente de subjuntivo, en el que únicamente aparece el radical procedente de VADERE en todas las personas. Por ello a continuación me ocupo tan solo de las tres formas que presentan variación en el radical: las formas correspondientes a vamos, vais $\mathrm{e} i \mathrm{~d}$.

Según Fernández Rei las variantes imos y vamos, así como ides y vades se registran en todo el territorio lingǘ́stico y a menudo existen las dos variantes en el mismo punto. La diferencia entre la cuarta y la quinta persona es que no hay zonas compactas en que aparezca solo una de las variantes de la cuarta, mientras se pueden determinar las zonas en que ides es más frecuente ${ }^{12}$. Las Normas ortográficas e morfolóxicas do idioma galego indican que las formas vamos y vades, que suelen ser consideradas castellanismos, fueron transferidas del presente de subjuntivo al presente de indicativo. La forma vamos es más frecuente en la lengua hablada que vades, por coincidir con la forma española. Por todo esto la lengua normativa da preferencia a imos e ides, mientras que vamos se acepta como forma de imperativo ${ }^{13}$. En lo que se refiere a las variantes de las tres formas, es interesante que Santamarina, en su libro esencial sobre el verbo gallego, en el paradigma

\footnotetext{
12 Francisco FERNÁNDEZ REI, Dialectoloxía da lingua galega, Vigo, Xerais, 1990, 97.

13 Real Academia Galega - Instituto da Lingua Galega, Normas ortográficas e morfolóxicas do idioma galego. A Coruña - Santiago de Compostela, Real Academia Galega - Instituto da Lingua Galega, $1995^{12}, 148$.
} 
del verbo ir indique imos, vamos; ides (iij), vades (vais); ide (ii) ${ }^{14}$. Es decir, mientras para la cuarta y la quinta persona del presente de indicativo da variantes con los dos radicales, para el imperativo únicamente indica variantes procedentes del latín IRE.

Según los datos del Atlas, para la cuarta persona del presente de indicativo tenemos dos variantes en el dominio lingüístico: imos y vamos (mapa 28515). Para la quinta persona del mismo tiempo encontramos ides, indes, ideis, is, vades, vandes, vais (mapa 286 ${ }^{16}$ ), es decir hay formas con los dos radicales. Además el sufijo también presenta variantes. Para el imperativo plural las variantes son ide, inde, $i$, ir, vade, vande (mapa 29217) (la forma ir es el infinitivo que aparece en función de imperativo plural). Por lo tanto en las tres formas tenemos variantes procedentes tanto de IRE como de VADERE. Vemos algunas asimetrías: no existe una forma como *idei, mientras encontramos ideis en el indicativo. Tampoco existe *vai, mientras se documenta vais. La no existencia de *vai tal vez se explica por el hecho de que con dicha forma se hubiera producido una colisión homonímica con la tercera persona del presente de indicativo.

Formas documentadas en el Atlas:

C1 imos/vamos - ides/vades - ide, C2 imos/vamos - vades - vade, C3 imos/vamos - ides/vades ide/vade, C4 vamos - ides/vades - ide, C5 imos/vamos - ides - ide, C6 imos/vamos - vades - ide, C7 imos/vamos - ides/vades - vade, C8 imos/vamos - ides/vades - ide/vade, C9 vamos - vades - vade, C10 imos - ides - ide, C11 imos - ides - ide, C12 imos-ides - ide, C13 imos/vamos - ides - ide, C14 imos/vamos - ides/vades - vade, C15 imos/vamos - ides/vades - ide, C16 imos/vamos - ides - ide, C17 imos/vamos - ides - ide, C18 imos - ides - ide, C19 imos - ides - ide, C20 imos-ides - ide, C21 imos - ides - ide, C22 imos - ides - ide, C23 imos/vamos - ides/vades - ide, C24 imos - ides/is - ide, C25 imos - ides - ide, C26 imos - ides-ide, C27 imos - ides - ide, C28 imos/vamos-ides - ide, C29 imos - ides - ide, C30 imos - ides - ide, C31 imos - ides-ide, C32 imos - ides - ide, C33 imos/vamos - ides - ide, C34 imos/vamos - ides - ide, C35 vamos - ides/vades - ide, C36 imos - ides - ide, C37 imos/vamos - ides - i, C38 imos - ides - ide, C39 imos - ides - ide, C40 imos - ides - ide, C41 imos ides - ide, C42 imos - ides - ide, C43 imos - ides - ide, C44 imos - ides - ide, C45 imos - ides - ide, C46 imos - ides - ide, C47 imos/vamos - ides - ide, C48 imos - ides - ide, C49 imos - ides - ide, P1 imos - ides - ide, P2 imos/vamos - ides - ide, P3 imos - ides - ide, P4 imos/vamos - ides - ide, P5 imos - ides - ide, P6 imos/vamos - ides - ide, P7 imos - ides - ide, P8 imos - ides - ide, P9 imos - ides - ide, P10 imos - ides - ide, P11 imos - ides - ide, P12 imos/vamos - ides - ide/ ir, P13 imos/vamos ides - ide, P14 imos - ides - ide, P15 imos/vamos - ides/vades - ide/ir, P16 imos/vamos - ides - ide, P17 imos - ides - ide, P18 vamos - ides/vades - ide, P19 imos - ides - ide, P20 imos/vamos ides/vades - ide/vade, P21 vamos - ides - ide, P22 imos - ides - ide, P23 imos/vamos - ides/vades $i d e / v a d e$, P24 imos/vamos - ides/vades - ide, P25 imos/vamos - ides - ide, P26 imos/vamos - ides ide, P27 imos - ides - ide, P28 imos/vamos - ides/vades/vandes - ide/vade/vande, P29 imos/vamos -

\footnotetext{
${ }^{14}$ Antonio SANTAMARINA, El verbo gallego, Santiago de Compostela, Universidade de Santiago de Compostela, Verba, Anexo 4, 1974, 93.

${ }^{15}$ Instituto da Lingua Galega, op. cit., 546.

${ }^{16}$ Instituto da Lingua Galega, op. cit., 548.

${ }^{17}$ Instituto da Lingua Galega, op. cit., 560.
} 
ides - ide, P30 imos/vamos - vades - ide, P31 imos - ides - ide, P32 imos - indes - inde, P33 imos/vamos - ides/indes - inde, L1 vamos - vades - vade, L2 imos/vamos - ides/vades - vade, L3 imos/vamos - ides - ide, L4 vamos - vades - vade, L5 imos/vamos - ides/vades - vade, L6 vamos ides/vades/vais - vade, L7 vamos - vades - vade, L8 vamos - vades/vai - vade, L9 imos - ides - ide, L10 imos/vamos - ides - ide, L11 imos/vamos - ides/vades - ide/vade, L12 imos/vamos - ides/vades - ide, L13 imos/vamos - ides - ide, L14 vamos - vades - vade, L15 vamos - vades - ide, L16 imos/vamos - ides/vades - ide, L17 imos - ides - ide, L18 imos/vamos - ides/vades - ide, L19 imos/vamos - ides/vades/vais - ide/vade, L20 imos/vamos - ides/vades - ide, L21 vamos $i d e s / v a d e s / v a i s$ - ide, L22 vamos - ides - ide, L23 vamos - ides - ide, L24 vamos - ides - ide, L25 vamos - vades - vade, L26 imos/vamos - ides/vais - ide, L27 vamos - ides - ide, L28 vamos - ides ide, L29 imos/vamos - ides/vades - ide, L30 imos/vamos - ides/vades - ide, L31 vamos - ides - ide, L32 imos/vamos - ides/vades - ide/vade, L33 imos/vamos - ides/vades - ide/vade, L34 vamos - ides - ide, L35 vamos - ides - ide, L36 vamos - ides - ide, L37 vamos - ides - ide/vade, L38 vamos - ides - ide, L39 vamos - ides/vades - ide, O1 imos - ides - ide, O2 imos/vamos - ides - ide, O3 imos/vamos - ides - ide, O4 imos - ides - ide, O5 imos - ides - ide, O6 imos - ides - ide, O7 imos ides - ide, O8 imos - ides - vade, O9 imos/vamos - ides - ide, O10 imos - ides/vades - vade, O11 imos - ides/vades - ide/vade, O12 imos - indes/vandes - inde, O13 imos - ides - ide, O14 imos - ides - ide, O15 imos/vamos - ides/vades - ide/vade, O16 imos/vamos - ides - ide, O17 vamos vades/vandes - vande, O18 imos/vamos - ides - ide, O19 imos - ides - ide, O20 imos/vamos - vandes - vande, O21 imos/vamos - vandes - ide/vade/vande, O22 imos/vamos - indes - inde, O23 imos ides / vades - ide, O24 imos - ides - ide, O25 imos - indes - inde, O26 imos - ides - ide, O27 imos ides - ide, O28 imos/vamos - ides - ide, O29 imos/vamos - ides - ide/vade, O30 imos - ides inde/vande, $\mathrm{O} 31$ imos/vamos - ides - ide/ $i$, A1 imos/vamos - ides/vades - ide, A2 vamos - ides/vades - ide, A3 imos/vamos - ides/vades - ide/vade, A4 imos/vamos - ides/vais - ide, A5 imos/vamos - ides - ide, A6 imos/vamos - ides/vais - ide/vade, A7 imos/vamos - vais - ide, Le1 imos/vamos - ides ide, Le2 imos/vamos - ides/vais - ide, Le3 imos/vamos - ides - ide, Le4 vamos - ides/vais - vade, Le5 vamos - ides/vais - vade, Z1 imos - ides - ide, Z2 vamos - ides/vais - ide, Z3 vamos - ideis - ide

En la cuarta persona imos se documenta en 64 puntos, vamos en 32 puntos, las dos formas a la vez en 71 puntos (en total hay 167 puntos dialectales). En la quinta persona del indicativo los números de ocurrencias son los siguientes (también tengo en cuenta la forma si solo aparece como facultativa): ides 147 , indes 5 , ideis 1 , is 1 , vades 49 , vandes 5 , vais 12. En el imperativo plural: ide 141, inde 6, i 2, ir 2, vade 33, vande 5 . Los números pueden engañar un poco, pues incluyen puntos en que la forma es la única existente y al mismo tiempo puntos en que conviven dos formas, p. ej. C9 vamos - vades - vade o C1 imos/vamos - ides/vades - ide. Según Ferreiro las formas vamos y vades casi siempre conviven con las formas imos e $i d e s^{18}$. Esta afirmación puede ser matizada: en el Atlas vamos aparece como forma única en 32 puntos, vades (o vais o vandes, es decir una forma procedente de VADERE) en 14 puntos.

A continuación observo la combinación de los radicales.

18 Manuel FERREIRO, Gramática bistórica galega, Santiago de Compostela, Edicións Laiovento, $1996,329$. 


\subsection{Radical}

El radical coincide en las tres formas:

IRE

p. ej. C10 imos - ides - ide, C24 imos - ides/is - ide

$\mathrm{C} 10, \mathrm{C} 11, \mathrm{C} 12, \mathrm{C} 18, \mathrm{C} 19, \mathrm{C} 20, \mathrm{C} 21, \mathrm{C} 22, \mathrm{C} 24, \mathrm{C} 25, \mathrm{C} 26, \mathrm{C} 27, \mathrm{C} 29, \mathrm{C} 30, \mathrm{C} 31, \mathrm{C} 32$, C36, C37, C38, C39, C40, C41, C42, C43, C44, C45, C46, C48, C49, P1, P3, P5, P7, P8, P9, P10, P11, P14, P17, P19, P22, P27, P31, P32, L9, L11, L17, L19, L25, L32, L33, O1, O4, O5, O6, O7, O13, O14, O19, O24, O25, O26, O27, Z1 (64)

(Las tres formas discrepan del resto de las formas de los presentes.)

VADERE

p. ej. C9 vamos - vades - vade, O17 vamos - vades/vandes - vande

C9, L1, L4, L7, L8, L14, L25, O17 (8)

(Todas las formas de los presentes tienen el mismo radical.)

IRE/VADERE

p. ej. C3 imos/vamos - ides/vades - ide/vade, A6 imos/vamos - ides/vais - ide/vade

C3, C8, P20, P23, P28, L19, L32, L33, O15, A3, A7 (11)

En los siguientes puntos hay diversidad de radicales:

cuarta persona ind. $\neq$ quinta persona ind. = imperativo plural

$\mathrm{P} 4$ ind. VADERE $\neq \mathrm{P} 5$ ind. IRE $=\mathrm{P} 5 \mathrm{imp}$. IRE

p. ej. C5 imos/vamos - ides - ide

C5, C13, C16, C17, C28, C33, C34, C37, C47, P2, P4, P6, P12, P13, P16, P21, P25, P26, P29, P33, L3, L10, L13, L22, L23, L24, L27, L28, L31, L34, L35, L36, L38, O2, O3, $\mathrm{O} 9, \mathrm{O} 16, \mathrm{O} 18, \mathrm{O} 22, \mathrm{O} 28, \mathrm{O} 31, \mathrm{~A} 5, \mathrm{Z3}$ (43)

(El sistema es como en portugués.)

$\mathrm{P} 4$ ind. IRE $\neq$ P5 ind. VADERE $=$ P5 imp. VADERE

p. ej. C2 imos/vamos - vades - vade

$\mathrm{C} 2, \mathrm{O} 11, \mathrm{O} 20(3)$

cuarta persona ind. $=$ quinta persona ind. $\neq$ imperativo plural

$\mathrm{P} 4$ ind. VADERE $=\mathrm{P} 5$ ind. VADERE $\neq \mathrm{P} 5$ imp. IRE:

p. ej. C1 imos/vamos - ides/vades - ide

C1; C15, C23, P15, P24, L12, L15, L16, L18, L20, L26, L29, L30, A1, A4 Le2 (16)

(El sistema es como en español.)

P4 ind. IRE $=$ P5 ind. IRE $\neq$ P5 imp. VADERE

p. ej. C7 imos/vamos - ides/vades - vade, O8 imos - ides - vade

$\mathrm{C} 7, \mathrm{C} 14, \mathrm{~L} 2, \mathrm{~L} 5, \mathrm{O} 8, \mathrm{O} 30$ (6)

No he tenido en cuenta los puntos dialectales en que aparece una combinación diferente, más dificil de describir, con soluciones facultativas para las formàs: $\mathrm{C} 4, \mathrm{C} 6$, 
C35, P18, P30, L6, L21, L37, L39, O10, O12, O21, O23, O29, A2, A7, Le4, Le5, Z2. P. ej. C4 vamos - ides/vades - ide, C6 imos/vamos - vades - ide. En el punto C4 la P4 del indicativo difiere en el radical del imperativo plural, la P5 del indicativo coincide con las otras dos formas; en C6 divergen la P5 del indicativo y el imperativo plural, mientras la P4 del indicativo coincide con las otras dos formas. Estos puntos son escasos y los modelos no pueden sistematizarse formando grupos.

Nuestra pregunta inicial era si además de los modelos esp. voy, vas, va, vamos, vais, van, id; port. vou, vais, vai, vamos, ides, vão, ide; gallego normativo vou, vas, vai, imos, ides, van, ide existen otras combinaciones posibles en el paradigma del gallego dialectal. Nuestra respuesta es básicamente negativa: el sistema mayoritario es el normativo, con las formas imos, ides; ide (para simplificar aquí no tengo en cuenta el sufijo de la quinta persona, la forma también puede ser $i$, indes, etc.). También es muy frecuente el sistema de tipo portugués: vamos, ides; ide, y existe asimismo el sistema español: vamos, vades; ide. Las otras combinaciones no son representativas.

\section{Las variantes dialectales correspondientes a VAMOS, VAIS e ID en catalán}

Solo pretendemos tratar este punto para comparar la gran variación dialectal del gallego con el caso catalán. Como hemos visto, las tres formas normativas del catalán central son ind. anem, aneu, imp. aneu.

Si observamos las variantes dialectales recogidas en La flexió verbal en els dialectes catalans de Alcover y Moll, podemos ver que prácticamente no hay variación dialectal en el radical. La forma anem tiene las variantes anam, nem, nam; de manera paralela las variantes de aneu (ind.) son anau, neu, nau, mientras aneu (imp.) posee las variantes anau, neu, nau y vaigau. La forma vaigau del imperativo es la única que procede de VADERE. Se documenta en un solo punto, el número 77 (Benavarri, catalán occidental). Esta forma es la del subjuntivo en este mismo punto dialectal (P4 vaigam, P5 vaigau, correspondientes a las formas normativas anem, aneu). En una serie de verbos catalanes, el imperativo, tanto en la segunda como en la quinta persona, adopta las formas del subjuntivo, p. ej. ser: sigues, sigueu; estar: estigues, estigueu; tenir. tingues, tingueu, etc. Siguiendo el modelo de estos verbos, en el punto 77 el imperativo plural del verbo anar adopta la forma del subjuntivo, es decir, vaigau. (Es interesante que en el imperativo singular no haya tenido lugar la misma sustitución: en el mismo punto dialectal la forma es ves, como en casi todo el dominio lingüístico, es decir la forma no procede del subjuntivo.) 


\section{Conclusión}

Los étimos que configuraron el paradigma del verbo IR y otros verbos iberorromances del mismo significado, así como la distribución concreta de los radicales, coincide a grandes rasgos en español, portugués, gallego, aragonés y asturiano, frente al francés y al italiano, que presentan características diferentes. El catalán en este caso discrepa de las otras lenguas iberorrománicas, pues su modelo es semejante al francés y al italiano.

Dentro del mismo tiempo verbal encontramos radicales no coincidentes para las diferentes personas gramaticales en el presente de indicativo y en el imperativo. Comparando el español, el portugués y el gallego, encontramos las siguientes combinaciones: esp. voy, vas, va, vamos, vais, van; id; port. vou, vais, vai, vamos, ides, vão; ide; gallego normativo vou, vas, vai, imos, ides, van; ide. Los tres paradigmas se explican fácilmente: o se distingue del resto de las personas el imperativo, o discrepan las dos quintas personas, o las tres formas que en los verbos regulares son arrizotónicas. La distribución de los radicales indica, pues, el grado de cohesión que existe entre las formas del paradigma. Hemos observado las formas dialectales del gallego, para comprobar si los sistemas muestran otras distribuciones de los radicales. Hemos podido comprobar que los paradigmas dialectales más frecuentes presentan la misma distribución que los tres modelos arriba mencionados. Los sistemas dialectales no son pues aleatorios: en ningún punto dialectal concuerdan elementos poco relacionados del paradigma, p. ej. no hay puntos en que la cuarta persona del indicativo y el imperativo plural tengan el mismo radical, frente a la quinta persona del indicativo (p. ej. imos-vadeside), a no ser con variantes facultativas. También llama la atención el hecho de que no haya variación dialectal en el radical del presente de subjuntivo, es decir, en todos los puntos dialectales el radical es uniforme en todas las personas. En efecto, en este tiempo la semejanza formal entre las seis personas es mucho mayor que en el presente de indicativo o entre las dos formas del imperativo (cf. verbos irregulares, p. ej. vexa, vexas, vexa, vexamos, vexades, vexan o la existencia de variantes dialectales rizotónicas de la cuarta y la quinta persona: cántemos, cántedes).

Las formas que discrepan de las otras personas del paradigma y que presentan variación dialectal en gallego son pues la cuarta y quinta personas del presente de indicativo y el imperativo plural. Las tres formas constituyen un grupo compacto en el paradigma verbal general por ser comunes en su estructura morfológica: constan de radical, vocal temática y sufijo número-personal (en un verbo regular cant-a-mos, cant-á-is, cant-a-d); son las formas arrizotónicas por excelencia en los presentes.

El catalán, en contraste con el gallego, prácticamente no hay variación dialectal en el radical en las formas que hemos examinado. 